\title{
Mind reading through the eyes: an fMRI study
}

\author{
Yujiro Kawata a c * , Eiji Kirino ${ }^{\mathrm{b}}$, Motoki Mizuno ${ }^{\mathrm{c}, \mathrm{d}}$ and Masataka Hirosawa ${ }^{\mathrm{c}, \mathrm{d}}$ \\ ${ }^{a}$ Tokyo Future University, Department of Child Psychology, Tokyo, Japan \\ ${ }^{\mathrm{b}}$ Juntendo University Shizuoka Hospital Department of Psychiatry, Shizuoka, Japan \\ ' Juntendo University School of Health and Sports Science, Chiba, Japan \\ ${ }^{d}$ Juntendo University Graduate School of Health and Sports Science, Chiba, Japan
}

\begin{abstract}
Mind reading is the ability to understand another person's thoughts, intentions, and feelings (Whiten, 1991). The purpose of this study was to clarify which part of the brain is evoked while mind reading in functional Magnetic Resonance Imaging (fMRI) experiments, using a mind reading task, the Reading Mind in the Eyes Test (RME: Baron-Cohen et al., 2001) with four choices (mental state words). The participants were right-handed and sex-matched healthy Japanese university students (10 males and 10 females, age range 19-25). We used periodic ABA...block design in the fMRI sessions. Task A (an experimental task) was the mind reading task and Task B (a control task) was the age-gender task. In order to clarify the activated region of the brain while mind reading, we calculated the difference between the degrees of BOLD (Blood-oxygen-level dependent) activation during the Task A and Task B. The results showed that there was a significant difference in activation of the right superior occipital gyrus, and the left parietal lobe. These findings indicated that these regions were used while mind reading. The role of these regions was discussed in terms of the findings from previous studies.
\end{abstract}

Keywords: Reading mind, Social cognition, Social brain, fMRI, BOLD

\section{Introduction}

Mind reading is the ability to understand another person's thoughts, intentions, and feelings [2]. This ability is so essential for successful social interaction that many researchers have paid much attention to it in terms of philosophy, primatology, developmental and social psychology, and cognitive neuroscience.

This ability is also called by several other terms: "theory of mind (ToM)" [3], "mentalising" [7], "social intelligence" [18], and "empathy" [9]. Also it is considered that this ability has evolved over time, especially in primates, because of selection pressure brought about by increasing social complexity. This hypothesis is called "social brain hypothesis" by Dunbar [15].

The origins of this ability are considered to be innately prepared [8]. Current hormone studies also support this idea, with evidence showing that the level of fetal testosterone (fT) is inversely correlated with the developing level of social behaviors shown by such indicators as eye contact [23], vocabulary level [22], and mind reading [4]. This ability is regarded as uniquely human and is considered to be an essential part of the defining the difference between humans and other species [16].

Also, it has been clarified that social impairments occur in some psychiatric disorders. For example, autism spectrum conditions [17], schizophrenia [10], and mood disorders [11] are included. Therefore, current numerous researchers pay much attention to these fields in order to clarify the social cognitive function.

So far, in order to assess the ability of social cognition, some psychological methods, for example, Theory of Mind Task or the Faces Test, have been developed [17]. Although these are useful for assessing the social ability of children or people with social impairments, they cannot be used to assess whether a normal adult has a mild or subtle social cognitive

\footnotetext{
* Corresponding author. E-mail: kawata-yujiro@tokyomirai.ac.jp
} 
deficit. They are too simplistic for normal adults to measure their social ability. Under these conditions, the Reading Mind in the Eyes Test was developed for use with adult persons with normal intelligence [20]. This test is able to measure mind reading among normal developed people. The RME consists of 36 eye-region photographs with four mental state words. Subjects are asked to choose the most adequate word which best describes the person's feeling and thinking exhibited in each photograph. The score is calculated by the sum of the correct answers. Nowadays this test is used even in some neuropsychological research using functional Magnetic Resonance Imaging (fMRI) $[13,18]$.

A neuro-physiological hypothesis of social cognition was first presented by Brothers [5], and she suggested that social cognition is involved in the following brain regions: the amygdala, orbitofrontal cortex (OFC), and superior temporal gyrus (STS). In addition, she postulated that the "social brain" consisted of these areas. Together, it was presented that these areas (amygdala, OFC, STS) were revealed to have a function in judging the others' expressed emotion, what is socially appropriate, and face-perception, respectively $([1,12,14])$.

However, recent studies have shown that other areas, such as inferior frontal gyrus (IFG), tomporoparietal junction, mid-temporal gyrus and etc., were also associated with mind reading [13, 19]. These indicate that there may be many other regions of the brain associated with mind reading. In addition, previous studies $[13,18]$ used the RME with only two choices of mental state words in the fMRI study in spite of the fact that the original RME has four choices of mental state words. Considering that mind reading is especially required in complex social situations, a more advanced-level test (with 4 mental state words to choose from) should be prepared.

The purpose of this study was to clarify which part of the brain is evoked while mind reading in fMRI experiments, using the Reading Mind in the Eyes Test with four mental state words choices.

\section{Methods}

\subsection{Participants}

The participants were right-handed and sexmatched healthy Japanese private university students (10 males and 10 females). Mean age was $22.0(\mathrm{SD}=$ 2.00) and age range was 19-25.

\subsection{Reading Mind in the Eyes Test}

We used the Reading Mind in the Eyes Test (RME) [20]. The RME is widely used for measuring the ability of facial affect recognition. This test can assess mind reading among normal developed people. The RME consists of 36 eye-region photographs with four mental state words (one correct word and three foil words). Subjects are asked to choose the most appropriate word which best describes the person's feeling and thinking exhibited in each photograph. A correct answer is scored 1 and a wrong answer is scored 0 . The total scores were calculated by adding up the number of correct answers. A higher score indicates better mind reading. The validity and reliability of the RME were exhibited in the original study [20].

\subsection{Procedure}

We used periodic ABA...block design in fMRI sessions using a 3 Tesra Philips system, Achieva Quaser Dual ${ }^{\circledR}$ with an 8 channel coil (SENSE-Head$8 \circledR)$. In high-resolution anatomical images, T1weighted (FFE-3D) sequence (TFE factor 141, NSA $=1, \mathrm{TR}=15 \mathrm{~ms}, \mathrm{TE}=3.5 \mathrm{~ms}$, Flip angle $=10^{\circ}, \mathrm{NEX}$ $=2$, slice thickness $=0.86 \mathrm{~mm}$, Slice\# $=190$, Matrix $364 \times 276$, direction sagittal, FOV $=$ FH $260 \times$ AP $236 \times$ RL $163 \mathrm{~mm}$, Voxel size $0.86 \times 0.86 \times 0.86 \mathrm{~mm}$ ) was employed. As functional images, a gradient-echo echoplanar sequence $(\mathrm{TR}=3000 \mathrm{~ms}, \mathrm{TE}=35 \mathrm{~ms}$, FOV $=25 \times 25 \mathrm{~mm}$, Slice Thickness $=6 \mathrm{~mm}$, Gap $=$ $0 \mathrm{~mm}$, Matrix $=96 \times 96$, Slice $=22$, Flip angle $=90^{\circ}$, EPI factor 95, Band Width in EPI $3037.3 \mathrm{~Hz}$ ) was employed. Event-related design $\{1 \mathrm{TR}=3 \mathrm{sec}$, SOA: 7-8TR (21-24s), Event : Rare, Baseline : Frequent, 1 block $=$ Total time $168 \mathrm{TR}(504 \mathrm{sec})=30$ events $\}$ was used for acquisition of fMRI data. The first 4 images were discarded to ensure steady-state longitudinal magnetization.

Each block was presented for 30 seconds, and there were six cycles of AB blocks in total. Task A (an experimental task) was the mind reading task composed of 36 photographs with 4 mental state words. Task B (a control task) was the age-gender task composed of 36 photographs with 4 age-gender words (young man, elderly man, young woman and elderly woman). The participants were asked to respond to each photograph using a right-handed button press (one of four buttons) during fMRI scanning. The SPM05 software was used for the preprocessing of fMRI images and voxel-based statistical analysis. 


\section{Results and Discussions}

In order to clarify the activated region of the brain while mind reading, we calculated the difference between the degrees of BOLD (Blood-oxygen-level dependent) activation during the Task A and Task B using the paired t-test. The results showed that there was a significant difference in activation of the right superior occipital gyrus and the left parietal lobe. These results indicated that these brain regions were characteristically used for mind reading.

The activations found in this study were basically consistent with the brain regions reported in previous fMRI studies using RME. Even when using RME with 4 choices task instead of the RME with 2 choices task, there was no remarkable difference. Thus, brain regions involved in mind reading may not be affected by the difficulty in the mind reading task.

\section{Conclusions}

From this study, it was concluded that the right superior occipital gyrus and the left parietal lobe are related to mind reading.

\section{Acknowledgements}

This work was supported by a grant from Juntendo University school of Health and Sports Science Campus-wide Collaborative Research and a grant from the Hi-tech Research Center at Juntendo University School of Health and Sports Science.

\section{References}

[1] A. Calder, A. Young, D. Rowland, D. Perrett, J. Hodges \& N. Etcoff, Facial emotion recognition after bilateral amygdala damage: Differently severe impairment of fear, Cognitive Neuropsychology 13 (1996), 699-745.

[2] A. Whiten, Natural Theories of Mind, Blackwell Publishing, Oxford, England, 1991.

[3] D. Premack and G. Woodruff, Does the chimpanzee have a "theory of mind"? Behavior and Brain Science 4 (1978), 515-526.

[4] E. Chapman, S. Baron-Cohen, B. Auyeung, R. Knickmeyer, K. Taylor, and G. Hackett, Fetal testosterone and empathy: evidence from the empathy quotient (EQ) and the "reading the mind in the eyes" test, Social Neuroscience 1 (2) (2006), $135-148$.

[5] L. Brothers, The social brain: a project for integrating primate behavior and neurophysiology in a new domain, Concepts in Neuroscience 1 (1990), 27-51.
[6] J. Flavell, F. Green, and E. Flavell, Development of knowledge about the appearance-reality distinction, Monographs of the Society for Research in Child Development 51 (1) (1986), 1-87.

[7] J. Morton, U. Frith, and A. Leslie, The cognitive basis of a biological disorder, Autism. Trends in Neuroscience 14 (1991), 434-438.

[8] M. Brune and U. Brune-Cohrs, Theory of mind: Evolution, ontogeny, brain mechanism and psychopathology, Neuroscience and Biobehavioral Revews 30 (2006), 437-455.

[9] M. Davis, Empathy -A Social Psychological Approach, Westview Press, Boulder, CO, USA 1996.

[10] M. Green, B. Olivier, J. Crawley, D. Penn, and S. Silverstein, Social cognition in Schizophrenia: Recommendations from the measurement and treatment research to improve cognition in schizophrenia new approaches conference, Schizophrenia Bulletin 31 (2005), 882-887.

[11] N. Krr, R. Dunbar and R. Bental, Theory of mind in bipolar affective disorders, Journal of Affective Disorder, 73 (2003), 253-259.

[12] P. Eslinger, \& A. Damasio, Severe disturbance of higher cognition after bilateral frontal lobe ablation: Patients EVR, Neurology 35 (1985), 1731-1741.

[13] R. Adams, O. N. Rule, R. Franklin, E. Wang, , M. Stevenson, , S. Yoshikawa., M. Nomura, , W. Sato, , K. Kveraga, and N. Ambady, Cross-cultural reading the mind in the eyes: an fMRI investigation. J.ournal of Cognitive Neuroscience 22(1) (2009), 97-108.

[14] R. Campbell, C. Heywood, A. Cowey, M. Regard \& T. Landis, Sensitivity to eye gaze in prosopagnosic patients and monkeys with superior temporal sulcus ablation, Neurophychologia 28 (1990), 1123-1142.

[15] R. Dunbar, The social brain hypothesis, Evolutionary Anthropology 6 (1998), 178-190.

[16] R. Saxe, and S. Baron-Cohen, Editorial: The neuroscience of theory of mind, Social Neuroscience 1 (2006), 1-9.

[17] Baron-Cohen, S. (1995) Mindblindness: An essay on autism and theory of mind. MIT press/Bradford Books, Boston.

[18] S. Baron-Cohen, H. Ring, S. Wheelwright, E. Bullmore, M. Brammer, A. Simmons and S. Williams, Social intelligence in the normal and autistic brain: An fMRI study, European. Journal of Neuroscience 11 (1999), 1891-1898.

[19] S. Baron-Cohen, H. Ring, X. Chitnis, S. Wheelwright, L. Gregory, S. Williams, M. Brammer, E. Bullmore, fMRI of parents of children with Asperger syndrome: A pilot study, Brain and Cognition 61 (2006), 122-130.

[20] S. Baron-Cohen, S. Wheelwright, J. Hill, Y. Raste, and I. Plumb: The "Reading the Mind in the Eyes" Test revised version: a study with normal adults, and adults with Asperger syndrome or high-functioning autism, Journal of Child Psychology and Psychiatry 42(2) (2001), 241-51.

[21] S. Baron-Cohen, T. Jolliffe, C. Mortimore, and M. Robertson, Another advanced test of theory of mind: Evidence from very high functioning adults with autism or Asperger syndrome. Journal of Child Psychology and Psychiatry 38 (1997), 813-822.

[22] S. Lutchmaya, S. Baron-Cohen, P. Raggatt, R. Knickmeyer and J. Manning, Fetal testosterone and vocabulary size in 18- and 24-month-old infants, Infant and Behavior Development 24 (2001), 418-424.

[23] S. Lutchmaya, S. Baron-Cohen, P. Raggatt, R. Knickmeyer, and J. Manning, Fetal testosterone and eye contact in 12thmonth-old infants, Infant and Behavior Development 25 (2002), 327-335. 